\title{
Evaluación de la Frecuencia, Ubicación y Tipos De Itsmos en Raíces Mesiobucales de Primeros Molares Maxilares. Un Estudio ex vivo
}

\author{
Evaluation of Isthmus Frequency, Location, and Types in \\ Mesiobuccal Roots of First Maxillary Molars. An ex vivo study
}

Daniela Matus* \& Mario Cantín ${ }^{*, * *}$

MATUS, D. \& CANTÍN, M. Evaluación de la frecuencia, ubicación y tipos itsmos en raíces mesiobucales de primeros molares maxilares. Un estudio ex vivo. Int. J. Morphol.,34(2):804-810, 2016.

RESUMEN: El objetivo fue describir la configuración interna del sistema canalicular de la raíz mesiobucal (MB) del primer molar maxilar, identificando el número de canales junto a la frecuencia, ubicación y tipos de itsmo a 1, 3 y 5 mm del ápice. Esta investigación fue aprobado por el comité de ética. Se realizó un estudio descriptivo ex vivo de corte transversal. Molares extraídos fueron limpiados, desinfectados y fijados en formalina al $10 \%$. Se identificó la raíz MB y se obtuvieron cortes transversales (3) a 1, 3 y 5 mm desde apical a coronal, identificadas como secciones A, B y C. Ciento cincuenta secciones fueron teñidas con azul de metileno y observadas bajo microscopio óptico, tanto en la superficie apical como coronal, y analizadas mediante el programa ImageJ. Se obtuvieron estadísticas descriptivas (media \pm DE), y mediante las prueba ANOVA y chi-cuadrado de Pearson se compararon las secciones a diferentes niveles. Se observaron un total de 445 canales radiculares, 289 correspondieron a canales accesorios. El 41,6 \% fueron canales mesiobucales secundarios (MB2) y 5,1 \% un tercer canal accesorio. Se observaron con mayor frecuencia istmos Tipo I (48), seguidos de Tipo IV (26); los Tipos II, III y V no superaron el $15 \%$. De acuerdo con la prueba de chi-cuadrado de Pearson, existen diferencias significativas entre los tipos istmos y distancia del ápice (p>0,001). La incidencia del Tipo I fue mayor cerca del ápice, con un descenso hacia los 3,0 mm. Se observó una compleja morfología del sistema canalicular apical en la raíz MB, que explica su baja tasa de éxito cuando son tratados con endodoncia. Las variaciones anatómicas, tales como MB2 o más canales accesorios e istmos deben ser considerados en la planificación y realización del tratamiento de endodoncia convencional o cirugía apical, con el fin de lograr procedimientos más exitosos. Se recomienda que las apicectomías se extiendan por lo menos a 3,3 mm del ápice. radicular:

PALABRAS CLAVE: Anatomía canalicular; Primer molar maxilar; Raíz mesiobucal; Canal mesiobucal; Istmo; Resección

\section{INTRODUCCIÓN}

El tratamiento de endodoncia tiene por objetivo la conformación, limpieza y obturación con un material de relleno inerte de todos los espacios de la cavidad dentinaria ocupada por el tejido pulpar (Cleghorn et al., 2006).

Conocer la morfología de los canales radiculares y sus frecuentes variaciones, es un requisito básico para el tratamiento endodóntico. En general se acepta que la mayor causa de fracaso en el tratamiento endodóntico es la incapacidad de reconocer y, por tanto, tratar adecuadamente todos los canales del sistema radicular (Verma \& Love, 2010).

Los primeros molares maxilares, en particular su raíz mesiobucal (MB), suelen ser tratados endodónticamente con una baja tasa de éxito (Imura et al., 1998). Estudios confir- man la compleja morfología de la raíz MB, reportando diversas variaciones anatómicas (Al Shalabi et al., 2000) tales como dos canales en más del $50 \%$ de los casos, itsmos o canales y foraminas accesorias (Donald et al., 1998). Betancour et al. (2013) reportan la presencia de un canal mesiobucal secuandario (MB2) en el 68,75\% de los casos, mientras que Hsu \& Kim (1997) una incidencia variable entre 5-53\% de istmos, la cual aumenta entre 3-5 mm de apical a coronal (Weller et al., 1995; Hsu \& Kim, 1997). Por su parte, Jung et al. (2005) demostraron que la prevalencia de canales accesorios e itsmos era mayor a los 2 y $5 \mathrm{~mm}$ apicales en la raíz MB. Esto permitiría explicar junto con la presencia de un segundo canal la alta tasa de fracaso terapéutico en este diente, ya que tanto los itsmos como los canales accesorios son áreas anatómicas imposibles de ser desbridadas

\footnotetext{
* Programa de Magíster en Odontología, Facultad de Odontología, Universidad de La Frontera, Temuco, Chile.

** Centro de Investigación en Morfología Aplicada (CIMA) \& CEMyQ, Facultad de Odontología, Universidad de La Frontera, Temuco, Chile.
} 
completamente de tejidos pulpares, restos necróticos y microorganismos (Somma et al., 2009). El conocimiento de esta complejidad en el tercio apical es importante de ser considerada tanto en el tratamiento de endodoncia como en la cirugía apical, evitando así el fracaso de ambos procedimientos (Jung et al.).

Los distintos porcentajes de canales MB2 reportados en la literatura pueden ser explicadas por las diferentes metodologías utilizadas para su detección. Buhrley et al. (2002) informaron de que la frecuencia de detección del canal MB2 en primeros molares maxilares al comparar el uso de microscopio vs. ningún tipo de aumento va desde $17,2 \%$ a un $71,1 \%$. Esto muestra que la experiencia del operador mejora como resultado del uso de un microscopio, y la prevalencia de la detección de canales accesorios aumenta al $93 \%$ (von Arx, 2005). Un estudio realizado por Baratto Filho et al. (2009) investigó la morfología interna de los primeros molares maxilares utilizando 3 métodos diferentes: ex vivo (usando un microscopio endodóntico para analizar dientes extraídos), clínica (usando un microscopio endodóntico combinado con registros radiográficos y fotográficos), y el análisis de CBCT, mostrando diferencias entre los métodos con respecto a la localización e identificación de los canales de la raíz, más fácil con el microscopio. En una revisión sistemática de la literatura, Betancourt et al. (2014) informan que los mayores porcentajes de detección del MB2 se obtienen mediante microscopios endodónticos: en estudios in vitro alcanzan el 92,3\%, mientras que en estudios in vivo el $73,2 \%$.

Con el advenimiento de los principios de microcirugía utilizando microinstrumentos y dispositivos de aumento (von Arx, 2005), un adecuado reconocimiento de la morfología del sistema canalicular y su configuración en el tercio apical, a nivel microscópico, sería un factor importante que podría mejorar la tasa de éxito en el tratamiento endodóntico y la cirugía perirradicular en dientes posteriores (Rubinstein \& Kim, 2002). Para Jung et al., la información sobre las variaciones anatómicas en secciones consecutivas de los ápices radiculares de molares puede ser de gran utilidad para los profesionales de endodoncia, especialmente durante la endodoncia quirúrgicas. Además, estudiar y observar de manera directa las estructuras de la región apical es relevante para el endodoncista, ya que durante el tratamiento endodóntico el clínico no observa de manera directa dicha región, relevando su importancia hacia un tipo posicional más que morfológico (Abarca et al., 2014).

El propósito de este estudio ex vivo fue investigar sobre una muestra de raíces MB de primeros molares maxilares, los tipos de configuración del sistema canalicular a 1, 3 y $5 \mathrm{~mm}$ de la región apical.

\section{MATERIAL Y MÉTODO}

El estudio fue aprobado por el Comité Ético Científico (CEC) de la Universidad de La Frontera (Protocolo 002/15). Se realizó un estudio descriptivo y observacional ex vivo de corte transversal, sobre la raíz mesiobucal de dientes primeros molares maxilares permanentes de ambos lados (16 y 26). Fueron incluidos dientes extraídos por indicaciones odontológicas, pertenecientes a sujetos de ambos sexos y mayores de 18 años, que poseían la raíz MB completa ( $\sin$ signos de radectomía o rizoclasia), con ápices totalmente formados, sin tratamiento endodóntico ni ortodóntico previo. Se excluyeron dientes con caries radicular, calcificaciones del sistema canalicular, fusión de la raíz MB y distobucal, evidencia de instrumentación o intervención odontológica a nivel de sus canales y presencia de dislaceraciones mayores a $30^{\circ}$.

Los molares extraídos fueron colocados y mantenidos en contenedores plásticos cerrados con formalina tamponada al $10 \%$, conservados bajo refrigeración. Posteriormente, cada diente fue sometido a una limpieza con puntas de ultrasonido Start-X® (Dentsply Maillefer, Ballaigues, Suiza) usando un equipo P5 Newtron ${ }^{\circledR}$ (Satelec, Acteon, Francia) para desbridar el tejido periodontal y cálculo, lavados con agua corriente y sumergidos en hipoclorito de sodio al $5 \%$ por $24 \mathrm{~h}$ con el fin de eliminar tejido orgánico residual. Luego, se lavaron nuevamente bajo agua corriente y se secaron.

En cada diente, se identificó la raíz MB y mediante un caliper se determinó su distancia a 1, 3 y $5 \mathrm{~mm}$ desde apical a coronal, obteniendo 3 secciones, A: desde el ápice a $1 \mathrm{~mm}$, B: desde el ápice a $3 \mathrm{~mm}$ y $\mathrm{C}$ : desde el ápice a $5 \mathrm{~mm}$ hacia coronal, respectivamente. Un investigador realizó previamente calibración para determinar las distancias. Luego, mediante un disco diamantado $(0,1 \mathrm{~mm}$ de grosor) montado sobre una pieza de mano, se cortó cada sección de la raíz, siguiendo las marcas realizadas bajo refrigeración con agua y aire, en un ángulo de $90^{\circ}$ respecto al eje longitudinal de la raíz (Fig. 1). Una vez obtenida las tres secciones de cada raíz, se mantuvieron en tubos eppendorf enumerados con formalina tamponada al $10 \%$ para impedir la deshidratación y el sobrecrecimiento bacteriano.

Las secciones fueron teñidas con azul de metileno para ser observadas bajo microscopio óptico Trinocular modelo CX31 (Olympus®, Hamburgo, Alemania). Cada sección (A, B y C) fue analizada tanto en su superficie apical (A1, B1 y C1) como coronal (A2, B2 y C2) (Fig. 1), obteniendo una muestra de 150 superficies. Se tomaron 


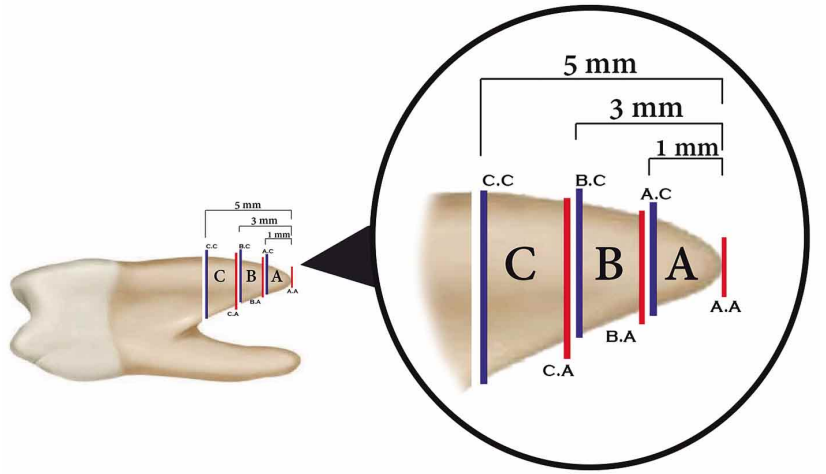

Fig. 1. Secciones obtenidas del tercio apical de la raíz MB del primer molar maxilar. Sección A a $1 \mathrm{~mm}$ del ápice, B a $3 \mathrm{~mm}$ del ápice y $\mathrm{C}$ a $5 \mathrm{~mm}$ del ápice a coronal. De cada sección se observó tanto la superficie apical (A.A, B.A y C.A) como coronal (A.C, BC y C.C) indicadas de color rojo y azul respectivamente.

fotografías con una cámara digital Moticam® ${ }^{\circledR} 480$ (Wetzlar, Alemania) integrada al microscopio, las que fueron analizadas con el programa ImageJ ${ }^{\circledR} 1.49$ para Mac OS X $(\mathrm{NIH}$ imagej.nih.gov/ij, USA). En cada sección, se determinó la presencia de canales MB1, MB2 y canales accesorios (o laterales). Además, se registró la presencia de istmos canaliculares y su tipo, utilizando la clasificación de Hsu \& Kim, donde el Tipo I fue definido como dos canales sin ninguna comunicación notable entre ellos; el Tipo II, como una conexión fina entre dos canales principales (MB1 y MB2); el Tipo III, igual que el Tipo II más la presencia de un tercer canal en lugar de dos; el Tipo IV, con un istmo con canales extendidos en la conexión y el Tipo V, reconocido como una verdadera conexión o amplio corredor entre los dos canales principales (Von Arx).

Análisis estadístico. Los datos fueron analizados mediante estadística descriptiva (Media \pm DE) y se utilizó la prueba ANOVA de un factor para relacionar las secciones observadas con el número de canales. La prueba de Chi-cuadrado se empleó para determinar si existían diferencias significativas entre los tipos de istmos y las secciones estudiadas. El análisis estadístico se realizó utilizando el programa SPSS/PC + versión 20.0 (SPSS, Chicago, IL, USA). Un valor de $\mathrm{P}>0,05$ fue elegido como nivel de significancia estadística.

\section{RESULTADOS}

Fueron analizadas 50 raíces MB, con 300 superficies obtenidas de las 150 secciones. La frecuencia de canales MB en los dientes observados fue de $26 \%, 62 \%$ y $12 \%$ para uno, dos y tres canales, respectivamente. En el total de las secciones, se observaron 445 canales radiculares, 289 correspondieron al tipo accesorio. El 41,6\% de los casos correspondió a la presencia de un canal MB2 y el 5,1\% a un tercer canal accesorio. La mayor cantidad de canales accesorios se observó a los 3,3 mm del ápice (Tabla I). Según la prueba de ANOVA de un factor, no existen diferencias significativas entre el número de canales y las secciones analizadas $(\mathrm{p}=0,717)$.

En las secciones, el $60 \%$ no presentó itsmos. Se observaron todos los tipos de itsmos según la clasificación de Hsu \& Kim (Fig. 2). La mayoría de los itsmos fue del Tipo I, con dos o tres canales sin comunicación definida. El Tipo IV fue el segundo más frecuente, donde los canales se extendieron al área del istmo, seguido de los Tipos II y V, evidenciando comunicación entre los canales (ver detalles en Tabla II). La cantidad de itsmos aumentó desde apical hacia coronal (1 a 28 itsmos). Según la prueba Chi Cuadrado de Pearson, existen diferencias significativas entre el tipo de itsmos y las secciones analizadas ( $p>0,001)$.

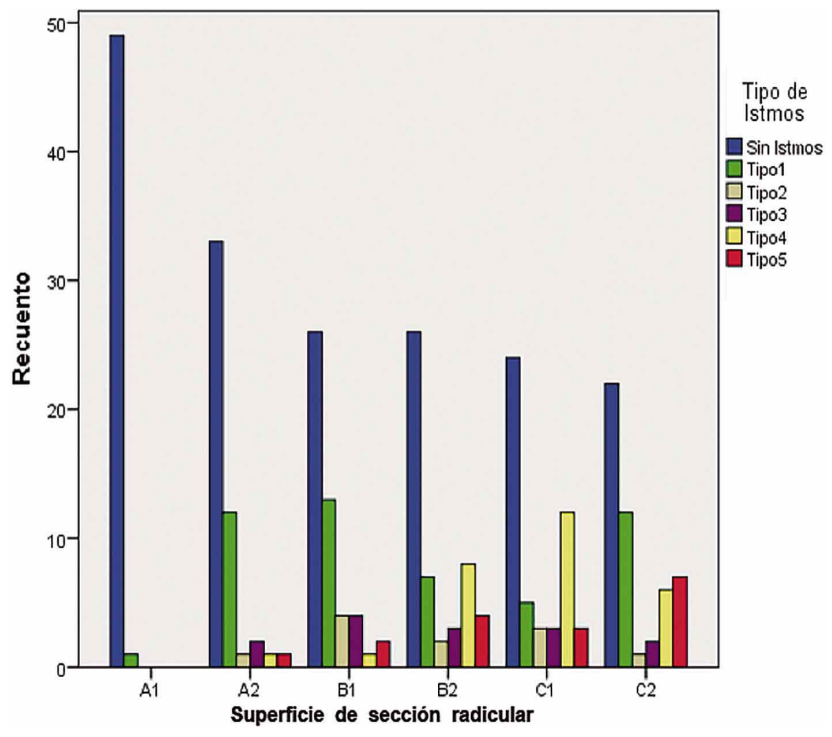

Fig. 2. Tipos de istmos observados en los diferentes niveles de sección. Tipo I: un, dos o tres canales principales redondeados $\mathrm{u}$ ovalados, pero sin formas de gota o señal de ramificación entre los canales. Tipo II: dos canales principales redondeados u ovalados, donde las paredes dentinarias entre los canales no alcanzan a fusionarse. Esta condición también se conoce como un istmo incompleta o parcial. Tipo III: similar al tipo II, excepto por la existencia de un tercer canal con apariencia de estar atrapado en la zona delgada de la raíz. Tipo IV: uno o dos canales caracterizados por una morfología en forma de gota o un itsmo parcial que disminuyendo en un istmo incompleto. Tipo V: reconocido como una verdadera conexión o amplio corredor entre los dos canales principales donde el tejido pulpar extiende a través de la parte delgada de la raíz y reconoce como un istmo completa o total. 
Tabla I. Frecuencia del número de canales radiculares en la raíz mesiobucal del primer molar maxilar permanente a diferentes distancias desde el ápice dentario.

\begin{tabular}{|c|c|c|c|c|c|c|c|c|}
\hline \multirow[b]{2}{*}{$\begin{array}{l}\text { Nivel de } \\
\text { sección }\end{array}$} & \multirow[b]{2}{*}{$\begin{array}{l}\text { Distancia } \\
\text { desde ápice }\end{array}$} & \multirow[b]{2}{*}{$\begin{array}{c}\mathbf{n} \\
\text { secciones }\end{array}$} & \multicolumn{3}{|c|}{ Número de canales } & \multirow{2}{*}{$\begin{array}{c}\# \\
\text { especímenes } \\
\text { con canales } \\
\text { accesorios }\end{array}$} & \multirow{2}{*}{$\begin{array}{c}\text { Total de } \\
\text { canales en } \\
\text { presencia de } \\
\text { canales } \\
\text { accesorios }\end{array}$} & \multirow[b]{2}{*}{$\begin{array}{l}\text { Total de } \\
\text { canales }\end{array}$} \\
\hline & & & 1 (MB1) & 2 (MB2) & 3 & & & \\
\hline A.A & $0 \mathrm{~mm}$ & $45^{*}$ & $23(51,1 \%)$ & $19(42,2 \%)$ & $3(6,7 \%)$ & $22(48,9 \%)$ & 47 & 70 \\
\hline A.C & $1 \mathrm{~mm}$ & $48^{* *}$ & $27(56,3 \%)$ & $20(41,6 \%)$ & $1(2,1 \%)$ & $21(43,7 \%)$ & 43 & 70 \\
\hline B.A & $1,3 \mathrm{~mm}$ & 50 & $27(54 \%)$ & $19(38 \%)$ & $4(8 \%)$ & $23(46 \%)$ & 50 & 77 \\
\hline B.C & $3 \mathrm{~mm}$ & 50 & $27(54 \%)$ & $21(42 \%)$ & $2(4 \%)$ & $23(46 \%)$ & 48 & 75 \\
\hline C.A & $3,3 \mathrm{~mm}$ & 50 & $26(52 \%)$ & $21(42 \%)$ & $3(6 \%)$ & $24(48 \%)$ & 51 & 77 \\
\hline C.C & $5 \mathrm{~mm}$ & 50 & $26(52 \%)$ & $22(44 \%)$ & $2(4 \%)$ & $24(48 \%)$ & 50 & 75 \\
\hline Total & & & 156 & 122 & 15 & 137 & 289 & 445 \\
\hline
\end{tabular}

*= En 5 secciones no se observó canal; **= En 2 secciones no se observó canal.

Tabla II. Tipo de itsmo y frecuencia de aparición en la raíz mesiobucal del primer molar maxilar permanente a diferentes distancias desde el ápice dentario.

\begin{tabular}{|c|c|c|c|c|c|c|c|c|}
\hline \multirow{2}{*}{$\begin{array}{l}\text { Nivel de } \\
\text { sección }\end{array}$} & \multirow{2}{*}{$\begin{array}{l}\text { Distancia } \\
\text { des de ápice }\end{array}$} & \multirow{2}{*}{$\begin{array}{l}\mathbf{n} \\
\text { secciones }\end{array}$} & \multirow[t]{2}{*}{ Sin itsmos } & \multicolumn{5}{|c|}{ Itsmos } \\
\hline & & & & Tipo I & Tipo II & Tipo III & Tipo IV & Tipo V \\
\hline \multirow[t]{2}{*}{ A.A } & $0 \mathrm{~mm}$ & 50 & 49 & 1 & 0 & 0 & 0 & 0 \\
\hline & & & $16,3 \%$ & $0,3 \%$ & $0,0 \%$ & $0,0 \%$ & $0,0 \%$ & $0,0 \%$ \\
\hline \multirow[t]{2}{*}{ A.C } & $1 \mathrm{~mm}$ & 50 & 33 & 12 & 1 & 2 & 1 & 1 \\
\hline & & & $11,0 \%$ & $4,0 \%$ & $0,3 \%$ & $0,7 \%$ & $0,3 \%$ & $0,3 \%$ \\
\hline \multirow[t]{2}{*}{ B.A } & $1,3 \mathrm{~mm}$ & 50 & 26 & 13 & 4 & 4 & 1 & 2 \\
\hline & & & $8,7 \%$ & $4,3 \%$ & $1,3 \%$ & $1,3 \%$ & $0,3 \%$ & $0,7 \%$ \\
\hline \multirow[t]{2}{*}{ B.C } & $3 \mathrm{~mm}$ & 50 & 26 & 7 & 2 & 3 & 8 & 4 \\
\hline & & & $8,7 \%$ & $2,3 \%$ & $0,7 \%$ & $1,0 \%$ & $2,7 \%$ & $1,3 \%$ \\
\hline \multirow[t]{2}{*}{ C.A } & $3,3 \mathrm{~mm}$ & 50 & 24 & 5 & 3 & 3 & 12 & 3 \\
\hline & & & $8,0 \%$ & $1,7 \%$ & $1,0 \%$ & $1,0 \%$ & $4,0 \%$ & $1,0 \%$ \\
\hline \multirow[t]{2}{*}{ C.C } & $5 \mathrm{~mm}$ & 50 & 22 & 12 & 1 & 2 & 6 & 7 \\
\hline & & & $7,3 \%$ & $4,0 \%$ & $0,3 \%$ & $0,7 \%$ & $2,0 \%$ & $2,3 \%$ \\
\hline Total & & $100 \%$ & $60,0 \%$ & $16,7 \%$ & $3,7 \%$ & $4,7 \%$ & $9,3 \%$ & $5,7 \%$ \\
\hline
\end{tabular}

Los tipos de istmo por nivel de sección se \&ïestra en la Figura 3. La incidencia de itsmos Tipo I fue mayor cerca del ápice, con una disminución evidente hacia los 3,0 mm, lo que sugiere un nivel de resección mínimo para procedimientos quirúrgicos u obturación retrógrada.

\section{DISCUSIÓN}

Las observaciones realizadas en este estudio en relación al número de canales de la raíz $\mathrm{MB}$ del primer molar maxilar fue de un $26 \%, 62 \%$ y $12 \%$ para uno, dos y tres canales respectivamente, estos valores difieren levemente de los encontrados por Degerness \& Bowles, quienes obtuvieron una frecuencia de $19,3 \%, 77,2 \%$ y $3,1 \%$ para uno, dos y tres canales. Esta variación se puede deber a que en el presente estudio se observaron los $5 \mathrm{~mm}$ apicales y no toda la longitud de la raíz $\mathrm{MB}$, junto a las posibles variaciones de la población estudiada, de sujetos chilenos. Por otro lado, si consideramos las secciones estudiadas el mayor número de canales se observó a los 3,3 mm del ápice y la frecuencia de dos y tres canales disminuye a un $41,6 \%$ y $5,1 \%$ respectivamente, lo que confirma la variable anatomía del tercio apical en donde los canales tienden a fusionarse o a desembocar a diferentes distancias del ápice anatómico. Esto además es apoyado por lo que observamos en relación a los itsmos, ya que en un $60 \%$ de las secciones no se registró su presencia, correspondientes a raíces $\mathrm{MB}$ con canal único. La observación microscópica coincide con lo observado por Reis et al. (2013) mediante CBCT, donde los canales MB2 son menos frecuentes en los tercios medio y apical que en el tercio coronal.

En las secciones estudiadas fue posible observar todos los tipos itsmos. Un istmo corresponde a una parte anatómica estrecha en forma de cinta que conecta o comunica 

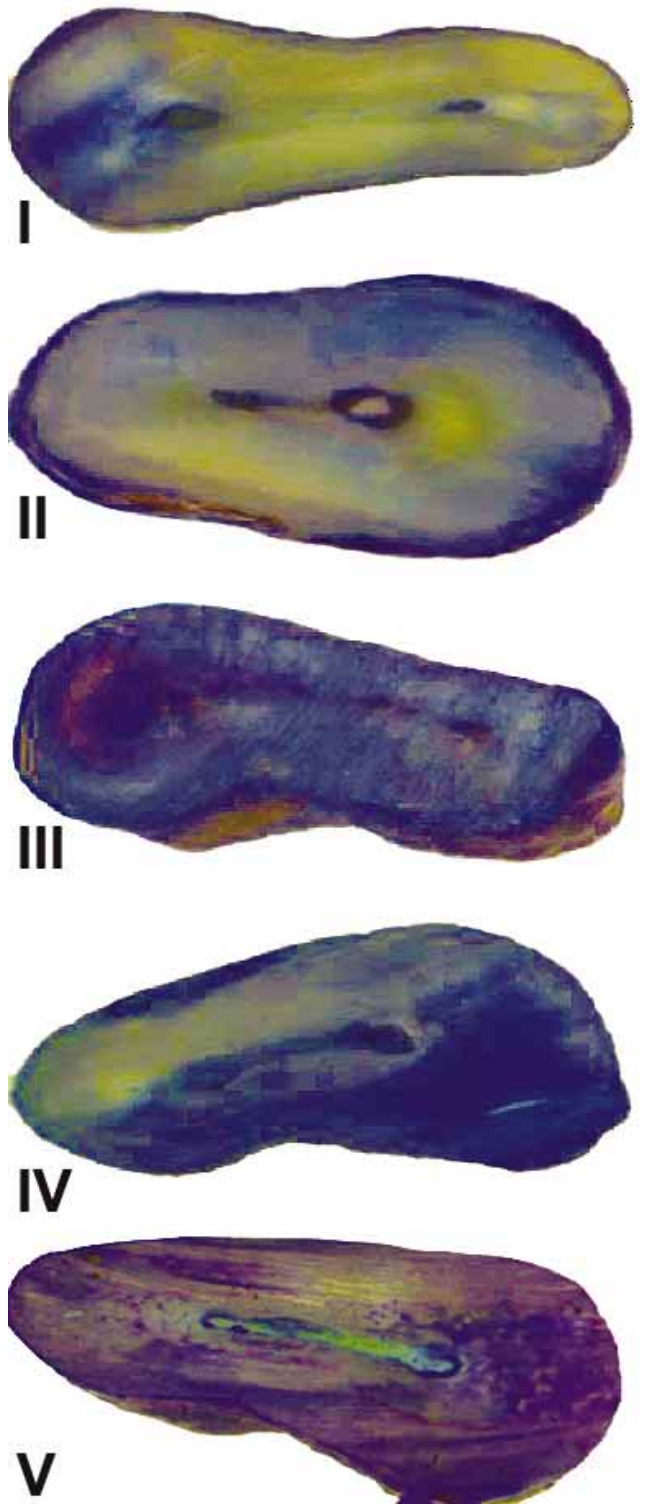

Fig. 3. Tipo de istmo por nivel de sección. La menor proporción de istmos se observan menos frecuentemente en la porción apical de la raíz. La incidencia de itsmos Tipo I fue mayor cerca del ápice y disminuyo progresivamente hasta los $3,3 \mathrm{~mm}$ desde el ápice, donde la incidencia aumentó hacia los $5 \mathrm{~mm}$. El itsmos Tipo IV aumentó desde los 1,3 mm hasta los 3,3 mm, y el Tipo V aumentó a medida que se acerco a la cámara pulpar (Tinción: Azul de metileno).

dos canales radiculares que contienen tejido pulpar (Weller et al., 1995), que pueden ser considerados como una conexión lateral entre canales de la misma raíz o clasificado como un anastomosis, razón por la que también se conoce como corredor o anastomosis transversal (Chai \& Tamse, 2015). Estos istmos se forman cuando una proyección radicular individual es incapaz de cerrarse completamente durante la odontogénesis, formando una constricción. La aproximación de las proyecciones radiculares pueden fusionarse por completo y formar una raíz con un canal radicular, como sucede con la raíz distobucal de los molares maxilares. Los resultados de fusión parcial dan lugar a la formación dos canales radiculares con un istmo entre ambos, como sucede en la raíz mesiobucal, mientras que la falta de fusión conduce a un canal en forma de cinta que contiene un istmo a lo largo de toda la raíz (Hsu \& Kim). Por otra parte, la deposición continua de la dentina por odontoblastos a lo largo de la vida también podría ser responsable de los diferentes tipos de istmos, lo que puede explicar los diferentes resultados reportados en la literatura actual (Lima et al., 2014).

La mayor incidencia de itsmos fue del Tipo I, con mayor cantidad en las secciones A.A, A.C y B.A, las más cercanas al ápice radicular. Los istmos Tipo I son definidos como dos canales sin comunicación, por lo tanto, de extenderse el canal MB2 a los último $3 \mathrm{~mm}$ de la raíz éste no tendría una comunicación con el MB1, haciéndose fundamental la limpieza, conformación y sellado de cada uno de los canales de manera independiente. A nivel de la sección B.C tanto el istmo Tipo I como IV fueron los más frecuentes obteniendo ambos valores similares, pero a nivel de la sección C.A, a 3,3 mm del ápice, el itsmo Tipo IV fue el más observado, situación que cambió a los $5 \mathrm{~mm}$ del ápice en donde el itsmo más frecuente fue nuevamente el Tipo I. Estos resultados se aproximan estrechamente a los obtenidos por Degerness \& Bowles quienes observaron una mayor frecuencia de canales accesorios y presencia de itsmos a los 3,6 mm del ápice, siendo los más frecuentes los Tipo I seguido del Tipo IV, lo que permite sugerir una relación inversa entre el número de canales y la presencia de itsmos Tipo I y un nivel mínimo de resección a los 3,3 mm apicales en la raíz MB del primer molar maxilar. La mayor frecuencia de los itsmos Tipos I también concuerda con lo reportados por Von Arx, quien reportó su presencia en el 37,5\% de la raíz MB del primer molar maxilar, siendo seguida por el Tipo III con un $31,2 \%$. Lyra et al. (2015) al evaluar raíces mesiobucales de 100 primeros molares maxilares reportaron que en cortes a los $3 \mathrm{~mm}$ sólo un $5 \%$ presentaban itsmos, y del Tipo I.

Clínicamente estos hallazgos son relevantes ya que el desbridamiento completo de estas áreas anatómicas mediante la preparación quimio-mecánica es complejo, y los tejidos necróticos y microorganismos de la pulpa pueden permanecer en la zona apical causando el fracaso del tratamiento endodóntico y provocando la necesidad de realizar una cirugía apical (Buhrley et al.). Recientemente, Kim et al. (2016) publicaron un estudio retrospectivo de molares sometidos a cirugía endodóntica, donde la tasa de éxito en dientes con itsmos está seriamente comprometida, con un riesgo de fracaso 6 veces mayor en los dientes que presentan itsmos en comparación con los dientes sin itsmos, concluyendo que es fundamental la investigación sobre la anatomía y presencia de istmos para cada diente.

Los dientes con itsmos Tipos II a V, en los que se observa claramente un istmo, deben ser preparados y obturados de manera retrógrada para eliminar las bacterias y bloquear la fuente de reinfección. En los Tipos IV y V, los clínicos pueden completar la preparación del extremo 
radicular lo largo del eje longitudinal de la raíz con movimiento pasivos de un dispositivo ultrasónico, debido a la clara estructura del istmo. Sin embargo, en los Tipos II y III, donde la estructura del istmo es menos clara, la ruta debe ser establecida activamente, donde puede ser difícil determinar con precisión la trayectoria apropiada y la angulación de la punta ultrasónica (Kim et al.).

Tradicionalmente la resección radicular se realizaba entre los 2-3 $\mathrm{mm}$ apicales de dientes tratados endodónticamente, sin embargo, el pensamiento actual es eliminar los $3 \mathrm{~mm}$ apicales de la raíz apoyado en estudios morfológicos (Degerness \& Bowles), y de acuerdo a los datos obtenidos por este estudio, la resección a los $3 \mathrm{~mm}$ apicales no sería suficiente, haciendo necesario extenderse a lo menos a 3,3 mm del ápice, logrando así exponer el área del istmo, eliminar complejidades del sistema de canales y permitiendo una correcta limpieza, conformación y obturación retrógrada de la zona, ya que un istmo sin preparación en el sistema de canales radiculares, especialmente en los dientes maxilares, podría contener restos necróticos y restos de tejido, lo que podría servir como un reservorio de bacterias, lo que lleva al fracaso endodóntico (Mehrvarzfar et al., 2014).

Los istmos canaliculares son a menudo desapercibidos y, si se encuentran, son de difícil instrumentación, donde movimientos pendulares de los instrumentos combinados con sustancias antimicrobianas y una abundante irrigación, optimizada con ultrasonido, son los procedimientos recomendados para mejorar la desinfección de la zona (Lima et al.). Si bien en la actualidad los clínicos cuentan con más y mejores herramientas, junto a tecnología que aumentan la calidad y tasa de éxito, como el microscopio que permite mejorar la visualización, así como técnicas imagenológicas avanzadas (radiografía digital o tomografía computadorizada que proporcionan información tridimensional), el conocimiento de la anatomía canalicular radicular es básico para compensar las barreras morfológicas del tratamiento endodóntico quirúrgico y no quirúrgico (Briseño-Marroquín et al., 2015).

Finalmente, esta información morfológica también es relevante para ser tomada en cuenta desde un punto de vista mecanicista, donde Chai \& Tamse establecieron que el istmo que conecta los canales radiculares puede ser considerado como un plano débil o grieta natural, donde una raíz mesial con dos canales radiculares es mucho más propensa a la fractura radicular vertical que la raíz con canal único, riesgo que aumenta durante la condensación de la gutapercha en las raíces con canales conectados por un istmo debido a las fuerzas de acuñamiento y la presión transmitidos a las paredes del canal, por lo que conocer el espesor de las paredes de estos canales con itsmos es un nuevo desafío para los clínicos.

\section{CONCLUSIÓN}

Las observaciones realizadas permiten una mejor comprensión del sistema de canales radiculares de la raíz mesiobucal del primer molar maxilar permanente. El conocimiento de sus frecuentes variaciones anatómicas, como el canal mesiobucal secundario, accesorios e itsmos, son factores importantes de ser considerados al momento de planificar y llevar a cabo tanto tratamientos endodónticos como cirugías periapicales en estos dientes posteriores, para lograr así procedimientos más exitosos. Se recomienda que las apicectomias se extiendan al menos a 3,3 $\mathrm{mm}$ del ápice en esta raíz.

MATUS, D. \& CANTÍN, M. Evaluation of isthmus frequency, location, and types in mesiobuccal roots of first maxillary molars. An ex vivo study. Int. J. Morphol., 34(2):804-810, 2016.

SUMMARY: The aim was to describe the internal configuration of the canalicular system of the mesiobuccal root (MB) of the maxillary first permanent molar, identifying the number of canals and isthmus frequency, location, and types at 1, 3 and $5 \mathrm{~mm}$ from the apex. This research was approved by the ethics committee. A descriptive ex vivo cross-sectional study was conducted. Extracted molars were cleaned, disinfected and fixed in 10\% buffered formalin. The non-instrumented MB root was identified and $3 \mathrm{cross}$ sections at 1, 3 and $5 \mathrm{~mm}$ from apical to coronal identified as A, B and C were obtained. 150 sections were stained with methylene blue and observed under microscope, in both apical and coronal surface using the ImageJ program. Descriptive statistics (Mean \pm SD) was obtained, and ANOVA and Pearson chisquare tests were used to compare the sections at different levels. A total of 445 root canals were observed, 289 corresponded to accessory canals. 41.6\% were secondary mesiobuccal canal (MB2) and 5.1\% a third accessory canal. The isthmus observed were Type I (48), followed by Type IV (26); Types II, III and V did not exceed 15\%. According to the Pearson chi-square test, there are significant differences between the isthmus types and distance of the apex ( $>$ > 0.001). The incidence of Type I was greater near the apex, with a decline towards 3.0 mm. A complex morphology of the apical maxillary MB root system was found, and explains their low success rate when treated endodontically. Anatomical variations such as MB2, accessory canals and isthmus should be considered when planning and conducting endodontic treatment or apical surgery, in order to achieve more successful procedures. It is recommended that apicectomies extend at least $3.3 \mathrm{~mm}$ from the apex.

KEY WORDS: Canal anatomy; Maxillary first molar; Mesiobuccal root; Mesiobuccal canal; Isthmus; Root resection. 


\section{REFERENCIAS BIBLIGRÁFICAS}

Abarca, J.; Zaror, C.; Monardes, H.; Hermosilla, V.; Muñoz, C. \& Cantin, M. Morphology of the Physiological Apical Foramen in Maxillary and Mandibular First Molars. Int. J. Morphol., 32(2):671-7, 2014.

al Shalabi, R. M.; Omer, O. E.; Glennon, J.; Jennings, M. \& Claffey, N. M. Root canal anatomy of maxillary first and second permanent molars. Int. Endod. J., 33(5):405-14, 2000.

Baratto Filho, F.; Zaitter, S.; Haragushiku, G. A.; de Campos, E. A.; Abuabara, A. \& Correr, G. M. Analysis of the internal anatomy of maxillary first molars by using different methods. J. Endod., 35(3):337-42, 2009.

Betancourt, P.; Fuentes, R.; Aracena, R. S.; Cantín, M. \& Navarro, C. P. Prevalencia del segundo canal en la raíz mesiovestibular de los primeros molares maxilares mediante tomografía computarizada de haz de cono. Av Odontoestomatol., 29(1):31-6, 2013.

Betancourt, P.; Cantín, M. \& Fuentes, R. Frecuencia del canal MB2 en la raíz mesiovestibular del primer molar maxilar en estudios in vitro e in vivo: una revisión sistemática. Av. Odontoestomatol., 30(1):11-22, 2014.

Briseño-Marroquín, B.; Paqué, F.; Maier, K.; Willershausen, B. \& Wolf, TG. Root Canal Morphology and Configuration of 179 Maxillary First Molars by Means of Micro-computed Tomography: An Ex Vivo Study. J. Endod., 41(12):2008-13, 2015.

Buhrley, L. J.; Barrows, M. J.; BeGole, E. A. \& Wenckus, C. S. Effect of magnification on locating the MB2 canal in maxillary molars. J. Endod., 28(4):324-7, 2002.

Chai, H. \& Tamse, A. The Effect of Isthmus on Vertical Root Fracture in Endodontically Treated Teeth. J. Endod., 41(9):1515-9, 2015.

Cleghorn, B. M.; Christie, W. H. \& Dong, C. C. Root and root canal morphology of the human permanent maxillary first molar: a literature review. J. Endod., 32(9):813-21, 2006.

Degerness, R. A. \& Bowles, W. R. Dimension, anatomy and morphology of the mesiobuccal root canal system in maxillary molars. J. Endod., 36(6):985-9, 2010.

Hsu, Y. Y. \& Kim, S. The resected root surface. The issue of canal isthmuses. Dent. Clin. North Am., 41(3):529-40, 1997.

Imura, N.; Hata, G. I.; Toda, T.; Otani, S. M. \& Fagundes, M. I. Two canals in mesiobuccal roots of maxillary molars. Int. Endod. J., 31(6):410-4, 1998.

Jung, I. Y.; Seo, M. A.; Fouad, A. F.; Spångberg, L. S.; Lee, S. J.; Kim, H. J. \& Kum, K. Y. Apical anatomy in mesial and mesiobuccal roots of permanent first molars. J. Endod., 31(5):364-8, 2005.

Kim, S.; Jung, H.; Kim, S.; Shin, S. J. \& Kim, E. The Influence of an Isthmus on the Outcomes of Surgically Treated Molars: A Retrospective Study. J. Endod., 42(7):1029-34, 2016.
Lima, F. J.; Montagner, F.; Jacinto, R. C.; Ambrosano, G. M. \& Gomes, B. P. An in vitro assessment of type, position and incidence of isthmus in human permanent molars. J. Appl. Oral Sci., 22(4):27481, 2014.

Lyra, C. M.; Delai, D.; Pereira, K. C. R.; Pereira, G. M.; Pasternak, J. B. \& Oliveira, C. A. P. Morphology of Mesiobuccal Root Canals of Maxillary First Molars: a comparison of CBCT scanning and Cross-sectioning. Braz. Dent. J., 26(5):525-9, 2015.

Mehrvarzfar, P.; Akhlagi, N. M.; Khodaei, F.; Shojaee, G. \& Shirazi, S. Evaluation of isthmus prevalence, location, and types in mesial roots of mandibular molars in the Iranian Population. Dent. Res. J. (Isfahan), 11(2):251-6, 2014.

Reis, A. G.; Grazziotin-Soares, R.; Barletta, F. B.; Fontanella, V. R. \& Mahl, C. R. Second canal in mesiobuccal root of maxillary molars is correlated with root third and patient age: a cone-beam computed tomographic study. J. Endod., 39(5):588-92, 2013.

Rubinstein, R. A. \& Kim, S. Long-term follow-up of cases considered healed one year after apical microsurgery. J. Endod., 28(5):37883, 2002.

Verma, P. \& Love, R. M. A Micro CT study of the mesiobuccal root canal morphology of the maxillary first molar tooth. Int. Endod. J., 44(3):210-7, 2011

von Arx, T. Frequency and type of canal isthmuses in first molars detected by endoscopic inspection during periradicular surgery. Int. Endod. J., 38(3):160-8, 2005.

Weller, R. N.; Niemczyk, S. P. \& Kim, S. Incidence and position of the canal isthmus. Part 1. Mesiobuccal root of the maxillary first molar. J. Endod., 21(7):380-3, 1995.

Weller, R. N.; Kimbrough, W. F. \& Anderson, R. W. A comparison of thermoplastic obturation techniques: adaptation to the canal walls. J. Endod., 23(11):703-6, 1997.

Dirección para Correspondencia:

Dr. Mario Cantín

Centro de Investigación en Morfología Aplicada (CIMA) \&

Centro de Excelencia en Estudios Morfológicos y Quirúrgi$\cos ($ CEMyQ)

Facultad de Odontología

Universidad de La Frontera

Pabellón L, Campus Andrés Bello

Av. Francisco Salazar 01145

Temuco - CHILE

Email: mario.cantin@ufrontera.cl

Recibido : 29-01-2016

Aceptado: 22-05-2016 